Muséologies

Les cahiers d'études supérieures

muséologies

\title{
Conception, évaluation et mise en oeuvre d'un programme éducatif de support à l'enseignement de l'écologie pour le collégial au Biodôme de Montréal : mission accomplie!
}

\section{Nathalie Matte}

Volume 1, numéro 1, octobre 2006

URI : https://id.erudit.org/iderudit/1033658ar

DOI : https://doi.org/10.7202/1033658ar

Aller au sommaire du numéro

Éditeur(s)

Association Québécoise de Promotion des Recherches Étudiantes en Muséologie (AQPREM)

ISSN

1718-5181 (imprimé)

1929-7815 (numérique)

Découvrir la revue

Citer cet article

Matte, N. (2006). Conception, évaluation et mise en oeuvre d'un programme éducatif de support à l'enseignement de l'écologie pour le collégial au Biodôme de Montréal : mission accomplie! Muséologies, 1(1), 64-79.

https://doi.org/10.7202/1033658ar d'utilisation que vous pouvez consulter en ligne. 
Conception, évaluation et

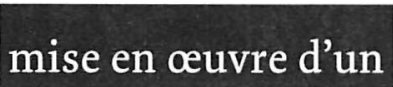

programme éducatif

de support à l'enseignement de

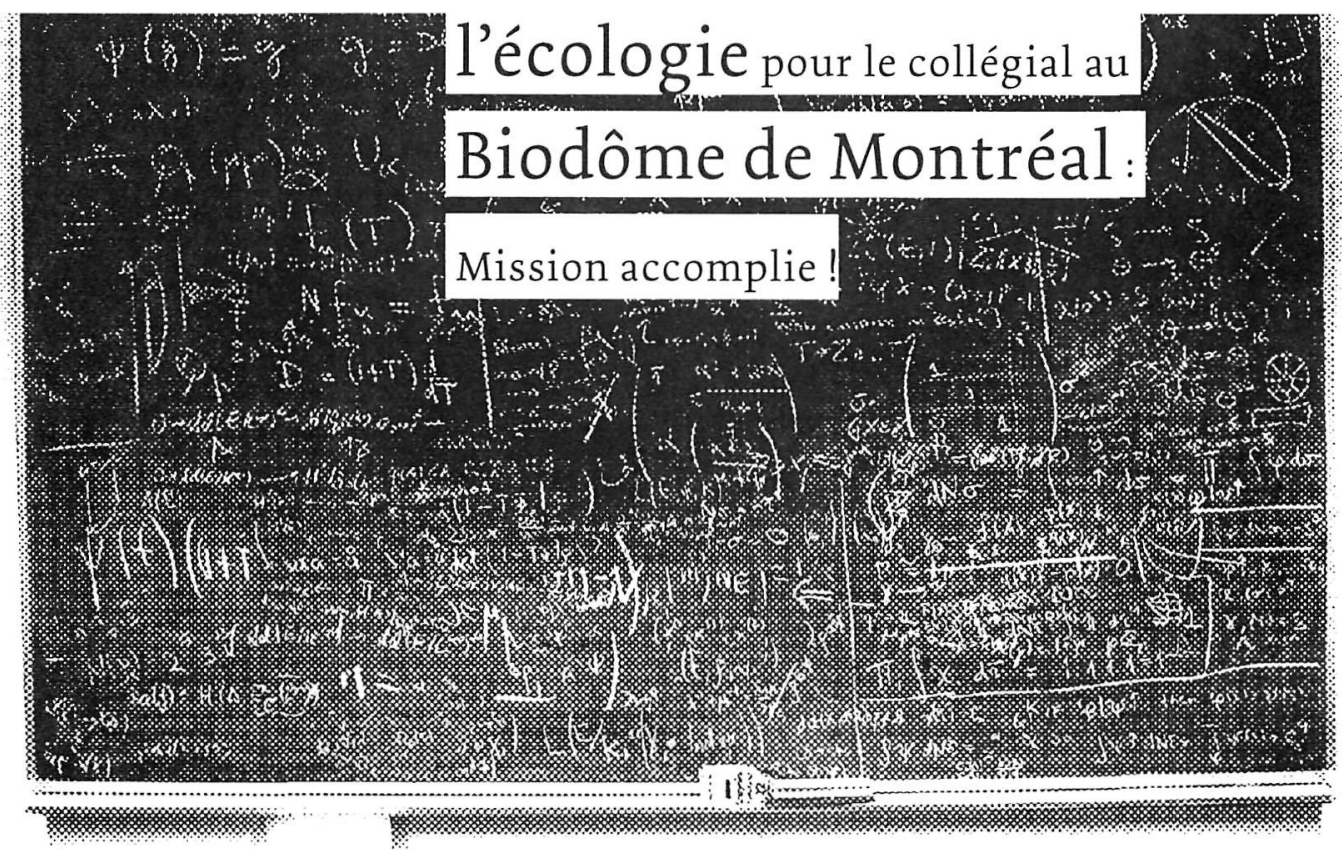

Nathalie Matte

Diplômée au baccalauréat en biochimie puis à la maîtrise en sciences appliquées au Département de génie chimique de l'École Polytechnique de Montréal, Nathalie Matte a choisi, après dix ans de pratique dans ce domaine, de réorienter sa carrière vers celui de la culture scientifique en complétant une maîtrise en muséologie à l'Université de Montréal en 2006. Elle est actuellement responsable de l'éducation au Musée Armand-

Frappier, Centre d'interprétation des biosciences. 
Résultat d'une collaboration entre Marie-Paule Otte, enseignante au collège Lionel-Groulx, le personnel du Biodôme et moi-même, ce projet dont l'originalité repose sur le choix du public qu'il sollicite a donné naissance au programme éducatif intitulé Mission intégration '"1. Présenté par M.-P. Otte lors du colloque collégial de l'Association des professeurs de sciences du Québec (APSQ) tenu à Montréal les 1er et 2 juin 2006, le programme a obtenu le prix de l'innovation pédagogique du concours Sortir des sentiers battus $2006^{[21}$.

La recherche dont cet article présente les faits saillants a permis de constater que les musées de sciences de la région de Montréal offraient peu d'activités à la clientèle des cégeps. Elle a permis d'identifier un intérêt de la part d'enseignants de biologie pour le programme développé, de compiler les perceptions d'une cinquantaine de cégépiens à propos du Biodôme et de voir l'enthousiasme de ces élèves pour une activité scolaire dans cette institution. De plus, le programme a suscité l'intérêt des animateurs en leur permettant d'approfondir les concepts écologiques lors des échanges avec les élèves.
L'auteure remercie Marie-Paule Otte et ses élèves du collège Lionel-Groulx, Claire Lépine, Nathalie Fleurent et Johanne Gravel ainsi que les animateurs et aquaristes du Biodôme de Montréal de leur participation à la recherche.

[2]

Le matériel pédagogique sera bientôt disponible sur le site de I'APSO: <www.apsq.org/ sautquantique/tres/ $\mathrm{t}$-scenarios6.html $>$ (consulté en juillet 2006) 
Biodôme. Mission. <www2. ville.montreal.qc.ca/biodome/ site/site.php> (consulté en septembre 2004)

[4]

LUSSIER-DESROCHERS, D.

et $T$. LEMERISE. "La relation

musées-adolescents démysti-

fiée». In. Lemerise, Tamara, Dany

Lussier-Desrochers et Victor Matias (dir.). Courants contemporains de recherche en éducation muséale. Québec : Éditions MultiMondes, 2002. p. 49-63

[5]

ALLARD, M. et S. BOUCHER Le musée et l'école. Montréal : Éditions Hurtubise $\mathrm{HMH}, 1991$.

136 p.; ALLARD, M. et S. BOUCHER. Éduquer au musée: Un modèle théorique de pédagogie muséale. Montréal Éditions Hurtubise HMH, 1998.

ALLARD, M. «Les adolescents et les musées *. Revue des sciences de l'éducation, 1993. vol. 1, no 4. p. 765-774; DIERKING, L.D., ELLENBOGEN, K M. et J. H. FALK. «In Principle, In Practice: Perspectives on a Decade of Museum Learning Research *. Science Education, 2004, vol. 88 (suppl. 1). p. S1S3; GIRAULT, Y. (dir.). L'accueil des publics scolaires dans les Muséums : Aquariums, Jardins botaniques, Parcs zoologiques.

Paris : L'Harmattan, 2003. 296 р. ; LUSSIER-DESROCHERS, D., LEMERISE, T. et 1 . LOPES. " Le groupe focus et le questionnaire : Deux méthodes complémentaires d'investigation des points de vue des adolescents sur les musées". In. LANDRY, Anik (dir.) en coll. avec Michel ALLARD. Le musée à la rencontre de ses visiteurs Québec : Éditions MultiMondes, 2003. p. 213-228; MATIAS, V et $T$. LEMERISE. "La relation musées-adolescents : L'importance du rôle des enseignants de l'ordre du secondaire." In. LEMERISE et al., op. cit., p. 67-87.

\section{Présentation du projet et intérêt pour la muséologie}

Enseignante d'expérience, M.-P. Otte déplorait l'absence de programmes muséaux s'adressant à ses élèves. En tant que future muséologue, je trouvais intéressant d'explorer un projet impliquant cette clientèle, peu connue des musées. À la suite de nos discussions, les objectifs de recherche suivants ont été définis :

- vérifier l'offre éducative des musées de sciences de la région de Montréal pour les cégépiens;

- vérifier l'intérêt des enseignants du collégial pour un programme de support à l'enseignement de l'écologie.

La consultation de l'énoncé de mission du Biodôme a permis de confirmer que ce lieu était idéal pour supporter l'enseignement de l'écologie ${ }^{[3]}$. Lors de discussions subséquentes, le personnel du Biodôme a attesté que les cégépiens qui le visitent sont peu nombreux. Ils choisissent parfois l'activité intitulée L'envers du décor qui explore les systèmes de support technique des écosystèmes.

Un préconcept, présenté à Claire Lépine, responsable des groupes scolaires au Biodôme, a ensuite été élaboré en scénario de programme. Les modalités d'expérimentation avec une classe ont été discutées et une personne-ressource a été mise à notre disposition. L'institution a accordé la gratuité d'accès à ses installations pour les élèves, l'enseignante et l'observatrice. À la suite de ces discussions, les autres objectifs visés par la recherche ont pris forme :

- tenter d'évaluer le potentiel de visiteurs que pouvait générer un tel programme;

- concevoir un programme réalisable qui respecte la mission du Biodôme, les objectifs du programme d'étude en écologie et les caractéristiques de la clientèle cible;

- déterminer les facteurs favorisant l'accès des cégépiens au Biodôme, par l'expérimentation du programme par des élèves et son évaluation.

La consultation de la littérature s'intéressant aux visites scolaires dans les musées a permis de constater que la nécessité de faire l'adéquation entre les objectifs du musée et ceux de l'école requiert un partenariat entre l'école et le musée afin de développer des services pertinents ${ }^{[4]}$. De plus, un des éléments à la base du succès des programmes éducatifs des musées est la connaissance des publics auxquels ils s'adressent ${ }^{[5]}$. Obtenir de l'information sur la clientèle cégépienne, peu connue du Biodôme, s’intègre ainsi aux objectifs de recherche.

Au moment de l'étude, aucune publication relative aux cégépiens en visite au musée n'a été identifiée. Plusieurs textes concernant les publics scolaires primaires et secondaires ont cependant été consultés. Ces publics, ainsi que les programmes qui leur sont offerts, y sont abondamment évalués ${ }^{[6]}$. Par ailleurs, 
la question des apprentissages y est un sujet récurrent ${ }^{[7]}$. Les apprentissages d'ordre affectif et social semblent plus importants que ceux réalisés sur le plan cognitif, puisqu'ils s'inscrivent, à long terme, dans le développement global de la personne ${ }^{[8]}$.

Trouvant dans ces recherches des arguments transposables au public collégial, nous avons choisi d'intégrer, en plus des objectifs d'évaluation des compétences disciplinaires en écologie ${ }^{[9]}$, des objectifs d'évaluation des compétences générales. Peu évaluées au collégial, il est essentiel de développer ces dernières tout au long des études préuniversitaires ${ }^{[10]}$.

Pour mieux connaître les élèves auxquels était destiné le programme éducatif, des publications sur la culture des jeunes ${ }^{[11}$ ainsi qu'une analyse statistique ${ }^{[121}$ ont été consultées. Celles-ci mentionnent que les jeunes souhaitent être actifs, participatifs et autonomes dans tous les domaines de leur vie et qu'ils étudient tout en travaillant. Conséquemment, ils ont peu de temps libre. Ces caractéristiques identifiées dans la documentation recensée ont été choisies et prises en compte dans l'élaboration du concept et du scénario du programme.

Afin de déterminer un scénario pertinent pour l'atteinte des objectifs pédagogiques, des articles concernant des programmes éducatifs particuliers ont été consultés. Selon les auteurs, la structure optimale comporte trois étapes : activité(s) préparatoire(s), réalisation de la visite au musée et activité(s) de consolidation post-visite ${ }^{(131}$. Cette structure a été adoptée pour scénariser Mission Intégration. Vérifier que cette structure permet de supporter l'enseignement de l'écologie s'est alors ajouté aux objectifs de recherche.

Cette recherche a donc été menée dans une optique de diversification des publics visés par une offre éducative spécifique, diversification que la politique muséale québécoise identifie comme un facteur pouvant contribuer à la vitalité de la muséologie actuelle ${ }^{[14]}$.

\section{Présentation du programme éducatif Mission Intégration}

La grille d'élaboration du programme éducatif préconisée par Allard et al. ${ }^{(15)}$ propose de définir les éléments suivants à l'étape de construction du scénario : public cible, objectifs, type d'animation pour chacune des activités, contenus notionnels, matériel, moyens, lieu et durée des activités. Le tableau 1 présente le détail de ces éléments.

La phase de préparation comprend quatre activités choisies en fonction d’objectifs pédagogiques, méthodologiques et logistiques.

La phase de réalisation comporte trois activités. Elle a comme point de départ la lecture d'une mise en situation fictive invitant les élèves à répondre à un

\section{[7]}

CASTEL, C. "Quelqu'un apprend-il quelque chose dans les musées canadiens ". Muse. 2005, vol. 23, no 5. p. 26-31.

[8]

FALK, J.H. "The Director's

Cut: Toward an Improved

Understanding of Learning from Museums". Science Education, 2004, vol. 88 (suppl. 1). p. S17S33.

[9]

Sur le plan disciplinaire, le programme Mission Intégration cible l'énoncé de compétence suivant : Analyser l'organisation du vivant, son fonctionnement et sa diversité et, plus spécifiquement, l'élément de compétence : Analyser l'intégration du vivant dans son milieu.

[10]

II s'agit des compétences suivantes : travailler en équipe, apprendre de manière autonome, utiliser les technologies de l'information et de la communication appropriées, communiquer de façon claire et précise et développer son sens critique.

[11]

VULTUR, M. «Les valeurs des jeunes et leur rapport aux institutions : Quelques pistes de réflexions pour l'intervention et la prévention». Conférence présentée au colloque Les jeunes et la SST dans une société en mouvement. Montréal, 28 avril 2005. 16 p.; Conseil de développement de la recherche sur la famille du Québec. «Qu'attend-on des jeunes aujourd'hui? Les jeunes et leurs valeurs". Compte rendu du colloque organisé en collaboration avec l'Observatoire Jeunes et Société. TroisRivières, 5 novembre 2004. $20 \mathrm{p}$. Ces documents sont disponibles sur le site de l'Observatoire Jeunes et Société de I'Institut national de la recherche scientifique / INRS-Urbanisation, Culture et Société : <www. obsjeunes.qc>. 
[12]

Collège Lionel-Groulx. Profil de la clientèle étudiante : Un portrait descriptif et statistique : 1998-2003. Enseignement régulier. Sainte-Thérèse, Collège Lionel-Groulx, 2004. 78 p. + annexes.

[13]

HENRIKSEN, E.K. et D. JORDE. «High School Students' Understanding of Radiation and the Environment: Can Museums Play a Role?n. Science Education, 2001, vol. 85. p. 189-206; ANDERSON, D., LUCAS, K.B. GINNS, I.S. et L.D. DIERKING

"Development of Knowledge about Electricity and Magnetism

during a Visit to a Science Museum and Related Post-Visit Activities". Science Education, 2000. vol. 84. p. 658-679.

[14]

Ministère de la Culture et des Communications du Québec. Politique muséale : Vivre autrement la ligne du temps. Québec : Publications du Québec, 2000. p. 26-27.

[15]

ALLARD, M., LAROUCHE, M.-C., MEUNIER, A. et P. THIBODEAU. Guide de planification et d'évaluation des programmes éducatifs. Montréal : Éditions Logiques, 1998. p. 101-104

[16]

CANTIN, R., LACASSE, D. et L. ROY. Intégration d'approches par problèmes en sciences de la nature, Phase I: Activité de synthèse. Rimouski : Cégep de Rimouski, 1996. 202 p. Ce document est disponible intégralement sur le site de l'APSO : $<w w w . a p s q . o r g / s a u t q u a n t i q u e /$ app/Rapport_Cegep_Rimouski. doc $>$ (consulté en juillet 2006). appel d'offres du Biodôme qui stipule que la direction de la recherche souhaite trouver des entreprises pour réaliser une analyse du potentiel d'intégration d'un nouvel animal dans un des écosystèmes. Après avoir généré une liste de critères caractérisant la niche écologique d'un animal, organisés en grille d'analyse comparative entre les milieux naturel et captif, les élèves déposent leur dossier de candidature. Les entreprises étudiantes reçoivent ensuite une lettre les informant que leur entreprise a obtenu le mandat. Un animal leur est attribué à partir d'une liste de douze animaux qui ont un potentiel d'intégration dans l'un des cinq écosystèmes.

Après une recherche documentaire préliminaire sur l'animal à intégrer, les entreprises étudiantes visitent le Biodôme de façon autonome. Cette visite leur permet de compléter la grille d'analyse du milieu captif. Le but de la démarche est de déterminer si l'animal peut ou non être éventuellement intégré à l'un des écosystèmes. Le dénouement du programme, en phase de consolidation, se fait par la présentation des résultats de recherche en classe.

Les phases de réalisation et de consolidation ont été pensées de manière à respecter l'approche de l'apprentissage par problème (APP) basée sur la théorie psychologique du constructivisme utilisée par les enseignants $\mathrm{du}$ collège Lionel-Groulx ${ }^{[16]}$.

[voir TABLEAU 1: Guide de référence du programme éducatif Mission Intégration, p. 69]

\section{Stratégie d'évaluation}

Nous avons élaboré une stratégie d'évaluation [voir TABLEAU 2: Stratégie et outils d'évaluation utilisés, p.72] afin de déterminer si les choix effectués étaient fondés, selon l'opinion des élèves, des enseignants et des animateurs, et aussi pour faire l'observation systématique de certaines activités.

Une expérimentation avait initialement été prévue. L'expérience du printemps 2005 ayant été perturbée par les grèves, M.-P. Otte a proposé de reprendre l'expérience à l'automne. Conséquemment, les étapes d'observation et d'administration des questionnaires sont différentes.

Cinquante-trois élèves du collège Lionel-Groulx ont évalué le programme pendant leur cours de biologie intitulé : Évolution et diversité du vivant ${ }^{[17]}$. Le questionnaire sommatif composé de 39 questions ouvertes et fermées a été administré aux élèves le dernier jour de l'expérimentation avec l'objectif de connaître leur opinion. Les habitudes socioculturelles des élèves ont fait l'objet d'une quinzaine de questions ouvertes et fermées réparties dans deux questionnaires préalables. Les observations en classe et au Biodôme ont été réalisées à l'aide de grilles choisies lors de la recension des écrits. Cependant, 
TABLEAU 1: Guide de référence du programme éducatif Mission Intégration ${ }^{[18]}$ [Phase de préparation] ${ }^{[19]}$

\section{Objectifs spécifiques Activité et objectifs}

Moyen

Lieu/par

Temps (min.)

- Préparer les élèves à l'objet d'étude : l'écologie

- Introduire les contenus notionnels

- Introduire les contenus notionnels

- Préparer les élèves à la cueillette de données

- Préparer les élèves à visiter le Biodôme
Déclencheur [Facultatif]

- Présenter le projet aux élèves

- Piquer leur curiosité

- Introduire le module d'écologie

- Explorer la notion d'écologie

- Générer une réflexion personnelle sur le développement durable

- Résumer par écrit un article de vulgarisation scientifique

- Exprimer son opinion

\section{Exploration du site Internet du Biodôme [Facultatif]}

- Consolider les notions d'écologie : écosystème

espèces, diversité biologique, extinction et population

Visite de la bibliothèque [Facultatif]

- Apprendre à faire une recherche bibliographique

- S'approprier des outils méthodologiques
- Lecture d'un article de vulgarisation scientifique sur la déforestation et le développe-

ment durable

- Résumé écrit de 1 page

- Schéma de concepts en écologie

- Retour en classe et débat

- Ordinateur(s) et/ou canon à projection, branchement Internet

Personnel de la bibliothèque
- Maison ou

bibliothèque/

Élèves

- Classe/

Enseignant

- Classe/

Enseignant et

élèves

- Classe, maison ou bibliothèque/

Enseignant et

élèves

- Maison ou bibliothèque

\section{[18]}

Durée totale du programme : 1 mois

[19]

Le choix des activités de la phase de préparation et de consolidation peut varier d'un enseignant à l'autre en fonction du temps et des moyens dont il dispose ainsi que de son intérêt personnel. Les objectifs spécifiques doivent cependant être couverts pour que les élèves puissent bénéficier du programme.

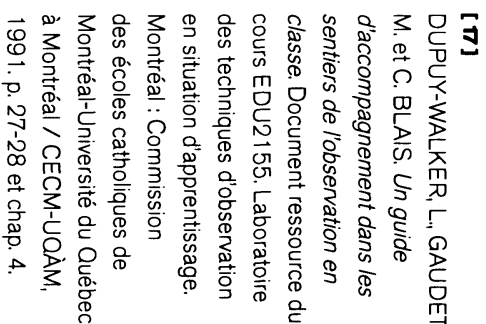


- Amener les élèves à découvrir le Biodôme, à le reconnaître comme lieu de conservation du patrimoine naturel et source d'information et de connaissances sur l'écologie

- Mettre en œuvre l'élément de compétence : analyse de l'intégration du vivant dans son milieu

- Solliciter et mettre en œuvre les compétences générales
Lecture de la mise en situation 1re partie [obligatoire]

- Former des équipes / Apprendre à travailler en

équipe, de manière autonome

- Définir des mots-clés / Clarifier les notions : écologie, espèce, écosystème, niche écologique, habitat, biodiversité, voie d'extinction

- Concevoir la grille d'analyse / Analyser un problème, appliquer la démarche scientifique, déterminer les critères qui permettent de comprendre l'intégration du vivant dans son milieu

Lecture de la mise en situation $2 e$ partie [obligatoire]

- Faire un retour sur la grille d'analyse / Valider la compréhension de la grille

- Rédiger une hypothèse concernant la localisation de l'organisme dans un des écosystèmes / Appliquer la démarche scientifique

- S'inscrire au calendrier des visites / Informer l'enseignant et le Biodôme des dates de visite

- Effectuer une recherche préliminaire / Trouver de l'information sur la niche écologique de l'animal

Visite au Biodôme [obligatoire]

- Admirer et observer des espèces animales vivantes dans des représentations d'écosystèmes nord-américains; identifier les caractéristiques des écosystèmes représentés; illustrer les relations obligatoires entre les organismes et leur milieu (adaptations physiques et physiologiques au milieu); identifier les possibilités et les limites techniques évidentes d'intégration d'un animal; localiser précisément l'endroit d'intégration de l'animal dans l'écosystème choisi

Compléter le dossier de recherche [obligatoire]

- S'assurer d'avoir tous les renseignements nécessaires à l'exercice de comparaison
- 1 copie du texte d'appel d'offres par élève

- Livre de référence Campbell, dictionnaire

- Assistance de l'enseignant

- 1 enveloppe-réponse par équipe

- Lettre-réponse avec en-tête du Biodôme

- Cahier du participant : consignes, dépliant du Biodôme, grille d'analyse corrigée, feuillet pour notes et schéma

- Visite des salles d'exposition du Biodôme en équipe

- Cahier du participant, tablette, crayons [obligatoire]

- Questions aux animateurs (en notant leur nom) [obligatoire]

- Audio-guide [facultatif]

- Observation avec des jumelles [facultatif]

Appareil photo [facultatif], sans flash [obligatoire]

- Livres, périodiques et ordinateur avec accès Internet
- Classe/

Enseignant et

élèves

- Classe,

maison ou

bibliothèque/

Enseignant et

élèves

\section{- Biodôme/ \\ Élèves}

\section{0}

temps

de visite)

- Maison ou
bibliothèque/
Élèves

(2h/élève) 
TABLEAU 1: suite [Phase de consolidation]

Objectifs spécifiques

- Permettre aux élèves d'analyser le résultat de

leurs recherches

- Amener les élèves à démontrer qu'ils ont compris l'importance des relations obligatoires entre les animaux et leur environnement, principalement la notion de niche écologique

- Solliciter les compétences générales

\section{Activité et objectifs}

Lieu/par

Temps

Mise en commun de l'information [obligatoire]

- Travailler en équipe

- Exercer son esprit critique

- Analyser les résultats de la recherche

- Confirmer ou infirmer l'hypothèse de départ

- Compléter la grille d'analyse de l'animal, le cas

échéant

- Finaliser le dossier de recherche

\section{Préparation d'une présentation orale en équipe ;}

validation du contenu de la présentation par les enseignants [Facultatif]

- Travailler en équipe

- Utiliser les TIC* appropriées

- Exercer son esprit critique

Présentation des résultats de la recherche aux

\section{autres élèves [Facultatif]}

- Permettre aux autres élèves d'explorer d'autres habi-

tats et de découvrir des animaux inconnus

- Permettre aux enseignants et aux élèves d'évaluer

l'ensemble de la démarche de recherche
Réunion d'équipe

- Cahiers du participant

Notes et références

Maison ou biblio-

thèque/ Élèves

Réunion d'équipe

- Cahiers du participant complétés

- Ordinateur avec le logiciel

PowerPoint

Présentation orale et support informatique

- Ordinateur avec le logiciel

PowerPoint

Classe/

- Diaporama de la présentation

- Canon

élèves

*Technologie de l'information et de la communication 
TABLEAU 2: Stratégie et outils d'évaluation utilisés

\section{Déroulement du programme}

Au préalable..

[20]

ALLARD, M. et B. LEFEBVRE. «Les habiletés intellectuelles mises en œuvre au musée". In. ALLARD et al. Évaluation et éducation muséale : Nouvelles tendances. 1998. p. 51-60;

Allard et al., Guide de planification..., op. cit., p. 159-165.

[21]

ALLARD et al., Guide de planification..., op. cit., p.186189; BORUN, M. et R. KORN (dir.). «Introduction to Museum

Evaluation». In. Professional

Practice Series. Washington : American Association of Museums, 1999. p. 1-51.

[22]

Collège Lionel-Groulx. Plan de cours : Bio-105-NYA. Évolution et diversité du vivant, 2004. 3 p.;

Ministère de l'Éducation, du Loisir et du Sport du Québec. $<w w w . m e l s . g o u v . q c . c a\rangle$. Discipline : biologie, code du cours : OOUK (correspondant au code

du cours NYA utilisé par les enseignants). p. 65 (consulté en novembre 2004).

Phase de préparation

- En classe

Phase de réalisation

- En classe

- Au Biodôme

\section{Phase de} consolidation

- En classe

\section{Groupe 1}

15 mai au 15 juin 2000

- Questionnaire Pour mieux se connaitre

- Questionnaire Projet de développement d'un programme éducatif en écologie
Ensemble du programme

- Grille d'observation des

- Grille d'observation des interactions en classe

- Grille d'observation des habiletés intellectuelles mises en œuvre au musée ${ }^{[21]}$

- Visites filmées par M-P. Otte

- Questionnaire d'opinion des animateurs (3)

- Grille d'observation des interactions en classe

- Grille d'évaluation pédagogique

- Grille d'évaluation par les pairs

- Grille d'autoévaluation (les élèves) Enseignants de LionelGroulx, Claire Lépine du Biodôme et Cécile Laurendeau
Groupe 2

6 septembre au 6 octobre 2000

- Questionnaire Pour mieux se connaître

- Les questions ont été intégrées au questionnaire sommatif interactions en classe ${ }^{[20]}$

- Ne s'applique pas

- Observateurs externes : Dubé du collège André-

- Ne s'applique pas

- Ne s'applique pas

- Visites observées et filmées par M-P. Otte

- Questionnaire d'opinion des animateurs (4)

- Ne s'applique pas

- Présentations filmées par M-P. Otte

- Grille d'évaluation pédagogique

- Grille d'évaluation par les pairs

- Grille d'autoévaluation (les élèves)

- Observatrice externe : Johanne Gravel du Biodôme

- Questionnaire sommatif aux élèves du groupe 2 
elles se sont révélées difficiles à utiliser dans le cadre de l'étude. L'utilisation d'une caméra vidéo a permis de revoir les interactions en post-observation et de confirmer celles qui ont prédominé. Sept animateurs ont répondu à un questionnaire d'opinion comportant dix questions dont trois ouvertes.

\section{Présentation des faits saillants}

L'offre éducative muséale en écologie pour le collégial

La consultation d'une trentaine de sites Internet d'institutions muséales de la région de Montréal a confirmé que l'offre éducative en écologie pour les élèves du collégial y était pratiquement absente. Seuls la Biosphère, L'Insectarium, le Musée Redpath, le Centre de la montagne du parc du MontRoyal, l'Écomuséum à Ste-Anne de Bellevue, le parc de la rivière des MilleÎles et le Musée Armand-Frappier ont pu être identifiés. Au moment où la consultation a été faite, aucun de ceux-là n'offrait d'activités spécifiquement conçues en fonction des objectifs pédagogiques du programme collégial d'écologie, les programmes de surveillance en nature de la Biosphère étant ceux qui touchaient de plus près les contenus notionnels.

Intérêt des enseignants de biologie du collégial pour un programme éducatif au Biodôme

La deuxième hypothèse de travail était qu'il existait un intérêt de la part des enseignants pour une activité de support à l'enseignement au Biodôme. Étant donné un contexte social défavorable ${ }^{[231}$, peu d'enseignants ont répondu au sondage acheminé par le biais d'un périodique électronique. Neuf enseignants ont répondu à l'appel et ceux du collège André-Grasset ont choisi d'expérimenter le programme avec quatre classes à l'automne 2005.

Une des interrogations du personnel du Biodôme concernait le potentiel de visiteurs que pourrait représenter le programme. Compte tenu de ce qui a été dit précédemment, il a été évalué que, pour le collège Lionel-Groulx, environ 200 élèves/année pourraient potentiellement y participer. Trois enseignants ${ }^{[24]}$ ont démontré un intérêt pour le projet, principalement parce qu'il s'agissait d'un projet intégrateur sur le plan des compétences disciplinaires et générales. Le sondage a été mené dans des conditions défavorables à une évaluation quantitative du potentiel de visiteurs que cette activité pourrait représenter. Il devrait être quantifié d'une autre manière, par exemple en contactant les coordonnateurs des départements de biologie.

\section{[23]}

Les enseignants du collégial étaient en négociation pour le renouvellement de leur convention collective.

[24]

Collèges Gérald-Godin, AndréLaurendeau et André-Grasset. 
Choix conceptuels et caractéristiques des elèves

Le concept développé mise sur une valeur actuelle du marché du travail, l'esprit d'entreprise, sur l'autonomie de la démarche et la participation des élèves. L'intérêt de connaître les profils socioculturels des élèves résidait dans l'absence de données les concernant dans la documentation en éducation muséale. Les données recueillies ont permis de prendre en compte certaines caractéristiques des élèves dans la structuration du programme éducatif. Les cégépiens ayant réagi très favorablement à la proposition qui leur a été faite, nous croyons qu'une partie de ce succès réside dans ce choix.

Les élèves interrogés avaient en moyenne 18 ans, occupaient un emploi à temps partiel de 12 à 14 heures par semaine, pratiquaient un loisir et suivaient six cours. En conséquence, ils n'étaient pas tous disponibles au même moment pour faire une activité. Le concept du programme a tenu compte de cette caractéristique en les laissant libres de visiter le Biodôme au moment qui leur convenait sur une période de dix jours. Plusieurs élèves ont mentionné avoir apprécié l'autonomie qui était attendue d'eux, alors que quelques-uns auraient apprécié davantage d'encadrement.

Les choix de carrière potentiels mentionnés par les élèves nécessitent, en majorité, une formation universitaire en sciences. Certains élèves ont souligné l'importance de travailler à développer des habiletés reliées au travail en équipe et à l'autonomie pour se préparer à l'université et au marché du travail. De plus, certains ont mentionné le plaisir de réaliser les activités proposées en équipe.

Plusieurs ont souligné l'importance des enjeux environnementaux relatifs aux forêts et la nécessité d'en discuter afin de trouver des solutions. Ils ont reconnu qu'ils trouvaient important d'exprimer leur opinion, de pouvoir la confronter à celle des autres et d'acquérir de nouvelles connaissances lors du débat. L'activité « déclencheur " répondait favorablement à ce besoin.

Les élèves avaient presque tous visité le Biodôme avant de faire ce projet et en avaient gardé un souvenir plaisant. Il importait donc de leur faire découvrir de nouvelles facettes de l'institution. Leur perception du Biodôme comme institution muséale était assez claire. Pour les uns le Biodôme est un lieu de conservation, d'exposition, de mise en valeur du patrimoine vivant et lieu d'apprentissage. Pour les autres, le Biodôme n'est pas un musée puisque ce dernier est associé exclusivement à l'art, à l'histoire, au passé, aux objets inanimés, au silence et à l'ennui. Il est heureux que plusieurs aient souligné le dynamisme du projet qui les invite à redécouvrir le Biodôme comme lieu de recherche et source de connaissances en écologie. 
Choix d'activités et opinion des elèves

Les choix d'activités ont, dans leur ensemble, plu aux participants. En phase de préparation, un texte sur les animaux ou les écosystèmes, un documentaire télévisuel ou un conférencier du Biodôme au collège auraient été appréciés.

En phase de réalisation, des améliorations pourraient être apportées aux textes de mise en situation, principalement l'appel d'offres, afin de préciser le sens du terme " grille d'analyse ". L'originalité des textes pourrait être travaillée avec des jeunes en groupe focus afin de savoir ce qu'ils trouvent amusant ou non. Au départ, nous craignions de découvrir que les élèves trouvent les textes insignifiants. Leur accueil enthousiaste lors de la remise des enveloppesréponses a désamorcé nos craintes. Les élèves ont fait la recherche documentaire préliminaire avec beaucoup de rapidité et de pertinence, triomphe d'Internet.

Une dimension d'authenticité a été introduite dans les activités afin de rendre le processus plus crédible : au Biodôme, la documentation des animaux dans un but d'acquisition se fait en considération des critères écologiques de la grille d'analyse ${ }^{[251}$, les animaux proposés appartiennent au plan de collectionnement ou ont déjà fait partie des collections, les documents utilisés par les élèves en portent le logo, la lettre est signée au nom d'une personne qui travaille au Biodôme. L'analyse des données a permis de constater que les élèves sont sensibles à cette dimension. Certains ont demandé s'il s'agissait d'un appel d'offres réel, si l'équipe choisie allait être payée pour faire le travail, si le personnel du Biodôme allait regarder les grilles d'analyse, etc. Lors de la visite, les élèves ont souligné leur intérêt à discuter avec des spécialistes, d'être pris au sérieux et non traités comme des "jeunes". Plusieurs ont découvert que les animateurs avaient une formation en sciences. Le manque d'animateurs sur les sentiers a été identifié comme la principale difficulté à la réalisation du projet.

L'observation des habitats, le questionnement et la communication entre les élèves et avec les animateurs ont été identifiés comme les quatre habiletés les plus souvent mises en œuvre par les participants des sept équipes. Les élèves ont été sensibles aux dimensions multiples de la visite. Certains ont dit souhaiter exercer un métier en relation avec les animaux, faire un stage au Biodôme, ont évoqué des souvenirs d'enfance reliés à la pêche, à la famille, souligné la dimension sensorielle de la visite (bien-être, température, odeurs, etc.) La visite de l'installation a été reconnue, par l'enseignante et les élèves, comme étant le cœur de ce projet en raison de l'expérience qu'elle procure. Démontrant leur intérêt, les équipes sont demeurées dans les écosystèmes de 90 minutes à trois heures.

\section{[25]}

Biodôme de Montréal, Muséums Nature de Montréal. Politique de gestion des collections vivantes, Copie de travail, document interne. 2005. 53 p. 
Opinion des animateurs

Les animateurs ont une opinion positive de la démarche utilisée, considérant que la comparaison entre les milieux naturel et captif est une façon efficace de prendre la mesure de la complexité des liens unissant le vivant à son milieu. Ils considèrent que le programme respecte la mission éducative du Biodôme. L'intérêt de la discussion aidant, le temps consacré aux équipes ne semble pas problématique. Étant donné la baisse d'achalandage du Biodôme en raison des moyens de pression des enseignants du primaire et du secondaire, les animateurs n'étaient pas particulièrement sollicités. De plus, ils ont dit qu'ils souhaitaient consulter la documentation produite concernant les animaux.

\section{Opinion de l'enseignante}

En phase de consolidation, bien qu'elles étaient dynamiques et soignées, l'enseignante a jugé que les présentations du groupe 1 ne ciblaient pas suffisamment l'élément d'analyse comparative. Le groupe 2 a mieux répondu aux consignes, pour la présentation orale et le dossier de recherche, l'enseignante ayant porté une attention particulière à ces aspects. Elle a avoué que le programme dépassait ses attentes relatives à la mise en œuvre des compétences générales.

\section{Conclusion : Mission accomplie!}

Mission Intégration est un programme éducatif muséal qui respecte la mission du Biodôme et les objectifs pédagogiques du cours d'écologie collégial puisqu'il a été élaboré en partenariat par le personnel enseignant et celui du Biodôme. La mise en œuvre du programme auprès d'élèves du collégial et son évaluation ont permis d'identifier les facteurs suivants, favorisant l'accès de ce public scolaire au Biodôme :

- peu d'institutions muséales de la région de Montréal offrent ce genre de programme, il y a donc un marché potentiel pour ce dernier;

- malgré un contexte social défavorable, des enseignants de collège ont manifesté leur intérêt pour utiliser le programme; il faudrait maintenant le publiciser et quantifier ce nouvel intérêt;

- valoriser les caractéristiques des jeunes de diverses manières;

- entretenir le partenariat avec les enseignants contactés;

- s'assurer de la disponibilité des animateurs sur les sentiers;

- favoriser la dimension d'authenticité de plusieurs manières.

En abordant l'étude de la clientèle scolaire collégiale, le projet a confirmé l'importance de connaître les caractéristiques de cette double clientèle à laquelle s'adresse le programme éducatif : les élèves et leurs enseignants. La recherche a permis de mieux connaître les élèves. La prise en compte de leurs 
caractéristiques est un facteur qui a favorisé l'accueil du programme. Il serait important de poursuivre le travail d'évaluation avec un plus grand nombre d'élèves et d'enseignants afin d'augmenter la pertinence du discours. Une attention particulière devrait maintenant être portée aux profils socioprofessionnels des enseignants et à l'organisation de l'enseignement collégial afin d'être en mesure de répondre plus adéquatement à leurs besoins.

Le travail de documentation des animaux est à poursuivre afin de diversifier les espèces offertes pour élargir le public, tout en soutenant l'intérêt des animateurs. Il importe de soutenir la dimension d'authenticité du programme afin d'assurer la crédibilité de la proposition auprès des jeunes. À ce niveau, l'expérience des animateurs et du personnel de recherche du Biodôme pourrait assurément être mise à contribution.

\section{Summary}

Mission accomplished! : Creation, evaluation and implementation of a CEGEP level educational program promoting the teaching of ecology at The Montreal Biodôme.

The originality of this educational program rests on the choice of the public targeted: CEGEP students. It is the result of a collaboration between Marie-Paule Otte, professor at Lionel-Groulx College, the staff at the Biodôme, and Nathalie Matte, author of this article.

This research has permitted us to conclude that science museums in the Montreal region offered few activities intended for cegep students. Research has also helped identify biology teachers' interest in the program that was developed. It compiled perceptions held by some fifty CEGEP students about the Biodôme and noted the interest and enthusiasm showed by these students in a field trip to the institution. The program elicited the participation of animators by permitting them to delve into ecological concepts during exchanges with the students.

\section{Presentation of the project and interest for museology}

The idea of such a program was born out of discussions had with Mrs. Otte. An experienced CEGEP professor, she deplored the lack of programs directed at her students. Following these discussions, the following research objectives were defined: 
- To verify the educational programs of Montreal science museums directed to CEGEP students

- To verify the interest of CEGEP biology teachers for a program that encourages instruction in ecology.

This research was led in order to diversify publics targeted by a specific educational offering. This type of diversification has been identified by Quebec museological policy as a factor contributing to the vitality of museology.

While consulting the mission statement of the Biodôme, we were able to confirm that this was an ideal place for promoting the teaching of ecology. During subsequent discussions, Biodôme staff attested that few CEGEP students visited the museum. They sometimes chose to visit L'envers du décor, an exhibit that presented technological systems of support for ecosystems. An early concept presented to Mrs. Lepine, the head of school groups at the Biodôme, was later developed into a program scenario. The modalities of experimenting with a class were discussed and a resource person was made available to us. The institution allowed free access to students, teacher and observer to its facilities. Following these discussions, other objectives identified by the researcher took shape:

- Evaluate the potential number of visitors that a program of the sort could bring to the Biodôme.

- Conceive a realistic program that respects the mission of the Biodôme, the objectives of the ecology study program and the characteristics of the targeted clientele.

- Determine which factors are favourable to CEGEP students to access the Biodôme through their experimentation of the program and the evaluation of their experiment.

Consultation of the literature dealing with school groups at museums allowed us to conclude that the necessity of positing a concordance between museum objectives and those of schools requires a partnership between schools and museums with a view to develop relevant services. Furthermore, knowledge of museum publics is one of the elements at the basis of museum educational programs' success. Obtaining information on CEGEP clientele, a group little known at the Biôdome, would become another research objective.

At the time of the study, no publication concerning CEGEP students who visit the Biodôme was identified. Many texts concerning primary and secondary school programs were consulted, however. These publics, as well as the programs that are offered to them, have been abundantly evaluated. The question of learning is a recurring theme.

In the long term, the emotional and social nature of learning appears to be more important than the cognitive level, as it has been inscribed in the global development 
of the individual. In finding arguments in these studies that can be transposed to the CEGEP public, we chose to integrate the evaluation of general qualifications in addition to the evaluation of core abilities in the field of ecology. Both are little evaluated at the CEGEP level; it is therefore essential to develop them throughout the entire course of pre-university studies.

To better understand the students for whom the educational program was destined, publications on youth culture as well as a statistical analysis were consulted. These noted that young people like to be active, participative, and autonomous in all areas of life. They study, work, and have little free time. These characteristics identified in the literature were chosen and taken into account in the development of the program concept and scenario.

In order to determine the appropriate structure for attaining the pedagogical objectives of the program, articles concerning particular educational programs were consulted. According to various authors, the optimal structure comprises three stages: preparatory activity (or activities), execution of the museum visit, and post-visit consolidation activity (or activities). This structure was used to write the screenplay of Mission Integration and it was also used to verify that it allowed another goal of the research: supporting the teaching of ecology. 